\title{
Whole tumor cell vaccines for glioma immunotherapy
}

\author{
"The development of high-throughput gene sequencing and \\ advanced bioinformatics has opened the exciting possibility \\ of identifying neoepitopes for personalized immunotherapy \\ in patients with cancer, including glioma."
}

First draft submitted: 14 December 2015; Accepted for publication: 19 December 2015; Published online: 14 March 2016

\section{Keywords: glioma $\bullet$ iNKT cells $\bullet$ vaccine}

High-grade glioma is associated with an extremely poor prognosis despite standard treatment with surgery, radiotherapy and chemotherapy. There is a pressing need to develop more effective treatments. Recent advances in immunotherapy for solid cancers have invigorated the search for an effective immunotherapy for glioma. There are many formidable obstacles such as the need to break tolerance to self-antigens and overcome tumor-induced immune suppression. Another fundamental problem is the fact that the optimum antigen targets for T-cell-based glioma immunotherapy remain unknown. Until recently, glioma immunotherapies reaching clinical translation have mainly targeted unselected antigens derived from autologous tumor tissue or defined selfantigens overexpressed in tumor relative to normal tissues (tumor-associated antigens [TAAs]). Theoretically, the ideal targets for cancer immunotherapy would be the products of tumor-specific mutations not previously encountered by the host immune system. Recent literature linking the success of checkpoint blockade in melanoma patients with responses directed at neoantigens has added weight to this idea. One described glioma neoantigen, EGFRvIII, is already at an advanced stage of assessment in clinical trials. However, with respect to glioma immunotherapy, the relative importance of targeting neoepitopes as opposed to TAA is not yet clear. Compared with other solid cancers, glioma harbors a relatively low num- ber of mutations, reducing the likelihood of immunogenic neoepitopes [1].

Other fundamental but unresolved questions concern the optimum number and $\mathrm{MHC}$ restriction of the target antigens. Gliomas are highly heterogeneous and genetically unstable so that targeting a single epitope can lead to antigen-loss and immune escape. A vaccine targeting an EGFRvIII epitope successfully eliminated EGFRvIII positive cells but did not prevent the recurrence of EGFRvIII negative tumors [2]. Although targeting a single specificity in association with a strong adjuvant can lead to epitope spreading, immunotherapies that target multiple specificities simultaneously seem more likely to succeed. With respect to $\mathrm{MHC}$ class restriction, it is now well established that $\mathrm{CD}^{+} \mathrm{T}$ cell help is necessary for a fully competent cytotoxic $\mathrm{CD}^{+} \mathrm{T}$ cell response. Indeed, there is some evidence that $\mathrm{CD} 4^{+} \mathrm{T}$ cells alone may be capable of mediating effective antitumor immune responses. This would suggest that the ideal cancer vaccine should incorporate both $\mathrm{MHC}$ class I and MHC class II-restricted epitopes.

Autologous tumor cells (ATC) have long been used as a source of antigens for incorporation into vaccines for glioma patients. ATC have the potential to provide a broad range of personalized tumor antigens that includes TAA and unique neoantigens, as well as epitopes for both $\mathrm{CD}^{+}$and $\mathrm{CD}^{+}{ }^{+} \mathrm{T}$ cells. Antigens derived from ATC have most commonly been loaded on to dendritic cells, which are then administered as a vaccine. However, the
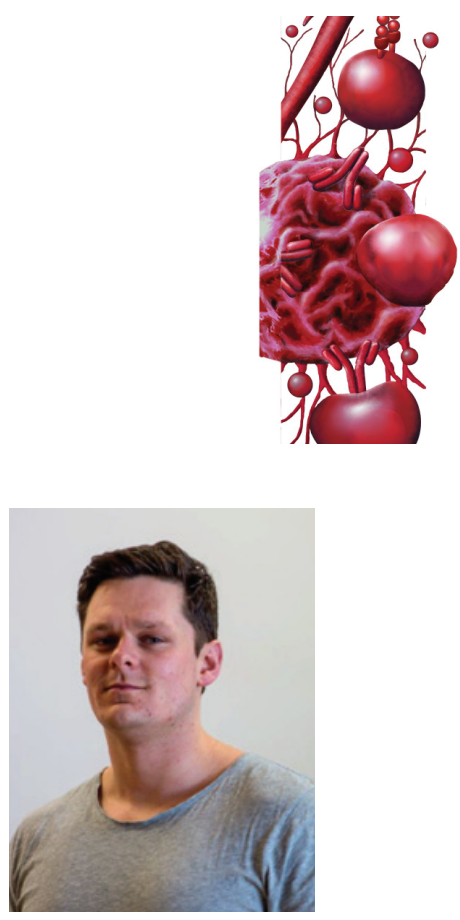

Cameron S Field

Malaghan Institute of Medical Research, Wellington 6242, New Zealand, and, School of Biological Sciences, Victoria University of Wellington 6242, New Zealand

\section{Ian F Hermans}

Malaghan Institute of Medical Research, Wellington 6242, New Zealand, and, School of Biological Sciences, Victoria University of Wellington 6242, New Zealand, and,

Maurice Wilkins Centre, Wellington, New Zealand

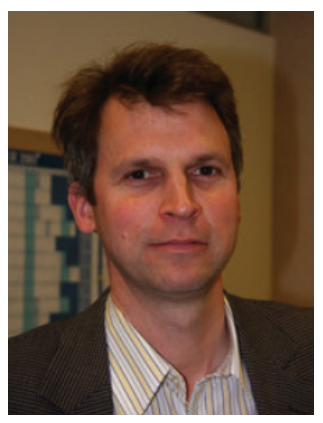

Martin K Hunn*

Malaghan Institute of Medical Research, Wellington 6242, New Zealand, and,

Capital \& Coast District Health Board, Wellington 6021, New Zealand

*Author for correspondence:

mhunn@malaghan.org.nz

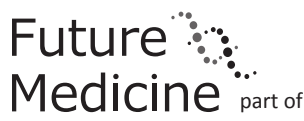


tumor cells themselves provide a ready-made vehicle for delivery of antigens to antigen-presenting cells (APCs) within the host.

We have previously explored the potential of a whole tumor cell vaccine comprised of irradiated murine glioma cells pulsed with the glycolipid $\alpha$-galactosylceramide ( $\alpha$-GalCer), a compound that stimulates type I invariant natural killer T cells (NKT cells). The glycolipid is acquired by host APCs and presented to NKT cells via the MHC class I-like molecule CD1d [3]; NKT cells recognizing their cognate antigen deliver a stimulatory signal through CD40-CD40L interactions that licences APCs to present tumor antigens to T cells. Since CD1d is nonpolymorphic and the transgenic $\mathrm{T}$ cell receptor of NKT cells is largely invariant, licensing by NKT cells is independent of tissue type, making $\alpha$-GalCer an attractive immune adjuvant with general utility. Pulsing tumor cells with $\alpha$-GalCer provides a platform that delivers antigen and adjuvant to the same APC, a phenomenon shown to be essential for inducing effective immune responses [4]. In this orthotopic murine model of glioma, we have previously demonstrated that the $\alpha$-GalCer-adjuvanted vaccine is capable of eradicating established intracranial tumors, but only if regulatory $\mathrm{T}$ cell (Treg)-mediated suppression is attenuated [5]. No strategy currently exists to specifically deplete Treg in human patients, leading us to explore other ways of enhancing vaccine efficacy in a therapeutic setting.

Recently, we have found that combining the vaccine with CTLA-4 blockade augments the antitumor immune response and leads to regression of established tumors in $80-100 \%$ of mice [Field $E T$ AL. Unpublished $\mathrm{DATA}_{\mathrm{A}}$. Therapeutic efficacy is associated with enhanced proliferation and accumulation of $\mathrm{T}$ cells in the lymphoid tissues without any obvious changes in the stimulatory function of APCs or the number of Tregs, suggesting recently primed $\mathrm{T}$ cells were the targets of checkpoint inhibition. While tumors regressing under this combined treatment were highly infiltrated with a variety of leukocytes, tumor eradication was strictly dependent on $\mathrm{CD}^{+} \mathrm{T}$ cells. These findings suggest that a vaccine consisting of ATC, pulsed with a powerful adjuvant and supplemented with checkpoint blockade, could be worth evaluating further in patients. The simple vaccine production method described in our studies could be adapted to generate a personalized ATC vaccine from resected tumor material in a short time frame, possibly requiring only $24 \mathrm{~h}$ in an GMP facility before administration. Alternatively, the vaccine could be cryopreserved until required.

In fact, a variety of ATC vaccines have already been tested in glioma patients. Nine clinical trials and two case reports of vaccination with ATC in 101 patients with high-grade glioma have been reported to date [6-16]. Note that this discussion does not include trials in which the main purpose of vaccination was to generate lymphocytes for adoptive T-cell transfer immunotherapy [17]. In most studies, tumor cells were irradiated prior to administration. In some, tumor cells were inactivated by exposure to chemotherapy or formalin fixation [10]. The ATC vaccines were administered subcutaneously or intradermally, with one exception in which irradiated ATC were enclosed in semipermeable diffusion chambers implanted temporarily in the rectus sheath [6]. Immunogenicity of the injected tumor cells has been enhanced in a variety of ways including admixture with fibroblasts engineered to release IL-2 [15] or IL-4 [12], continuous infusion of GM-CSF at the vaccination site [7], coadministration with BCG [10] or infection of the tumor cells with Newcastle virus [14,16]. In other studies the tumor cells were engineered prior to injection, to express proinflammatory cytokines and costimulatory molecules [13], to downregulate expression of the immunosuppressant cytokine TGF- $\beta 2$ [8] or to downregulate expression of Insulin-like growth receptor-1 which in turn induces tumor cell apoptosis [6]. The vaccines were well tolerated and the results of these studies have been broadly similar to clinical studies of DC vaccines with most reporting objective responses in a minority of patients and/or a survival benefit in comparison to historical controls. It is likely that these results could be significantly improved by incorporating recent advances in the field. Importantly, the often complex methods used to enhance immunogenicity of the tumor cells meant that significant feasibility issues were encountered in several of these studies [7,12]. The simple vaccine production method described here would avoid these difficulties.

A valid concern about ATC vaccines is the risk of side effects from targeting self-antigens. Lethal allergic encephalomyelitis has been reported in rats vaccinated with human glioblastoma multiforme tumor cells [18]. However, no adverse events attributable to autoimmunity were reported in any of studies of ATC vaccines in human patients, including one study that specifically assessed humoral responses to myelin basic protein and evidence of demyelination on MRI [7]. Another issue is the route of administration. While animal studies have shown that glioma cells can be highly immunogenic when injected subcutaneously [19], it has been reported that $T$ cells become licensed to enter the central nervous system by prior transit through the lung [20]; it is possible that this may be more readily achieved by intravenous vaccine administration. In our studies, where the vaccine was administered intravenously to optimize access to NKT cells, we noted some coagulation-related toxicity, which could be completely abrogated by pretreatment with heparin. Nonetheless, we suggest that further 
studies are required to optimize efficacy and safety with respect to route of administration.

In summary, we have described a simple ATC-based vaccine in a glioma model that holds promise for clinical translation. The development of high-throughput gene sequencing and advanced bioinformatics has opened the exciting possibility of identifying neoepitopes for personalized immunotherapy in patients with cancer, including glioma. A clinical trial based on this approach in GBM patients is already underway (NCT02149225). However, the method is complex and time consuming and feasibility is yet to be established. In the meantime, ATC remain attractive as a ready-made source of personalized antigens that can also serve as a physical platform for efficient delivery

\section{References}

1 Schumacher TN, Schreiber RD. Neoantigens in cancer immunotherapy. Science 348(6230), 69-74 (2015).

2 Sampson JH, Archer GE, Mitchell DA, Heimberger AB, Bigner DD. Tumor-specific immunotherapy targeting the EGFRvIII mutation in patients with malignant glioma. Semin. Immunol. 20(5), 267-275 (2008).

3 Kawano T, Cui J, Koezuka Y et al. CD1d-restricted and TCR-mediated activation of valpha14 NKT cells by glycosylceramides. Science 278(5343), 1626-1629 (1997).

4 Farrand KJ, Dickgreber N, Stoitzner P, Ronchese F, Petersen TR, Hermans IF. Langerin + CD $8 \alpha+$ dendritic cells are critical for cross-priming and IL-12 production in response to systemic antigens. J. Immunol. 183(12), 7732-7742 (2009).

5 Hunn MK, Farrand KJ, Broadley KW et al. Vaccination with irradiated tumor cells pulsed with an adjuvant that stimulates NKT cells is an effective treatment for glioma. Clin. Cancer. Res. 18(23), 6446-6459 (2012).

6 Andrews DW, Resnicoff M, Flanders AE et al. Results of a pilot study involving the use of an antisense oligodeoxynucleotide directed against the insulin-like growth factor type I receptor in malignant astrocytomas. J. Clin. Oncol. 19(8), 2189-2200 (2001).

7 Clavreul A, Piard N, Tanguy JY et al. Autologous tumor cell vaccination plus infusion of GM-CSF by a programmable pump in the treatment of recurrent malignant gliomas. $J$. Clin. Neurosci. 17(7), 842-848 (2010).

8 Fakhrai H, Mantil JC, Liu L et al. Phase I clinical trial of a TGF-beta antisense-modified tumor cell vaccine in patients with advanced glioma. Cancer Gene Ther. 13(12), 1052-1060 (2006).

9 Ishikawa E, Tsuboi K, Yamamoto T et al. Clinical trial of autologous formalin-fixed tumor vaccine for glioblastoma multiforme patients. Cancer Sci. 98(8), 1226-1233 (2007).

10 Muragaki Y, Maruyama T, Iseki H et al. Phase I/IIa trial of autologous formalin-fixed tumor vaccine concomitant with fractionated radiotherapy for newly diagnosed glioblastoma. Clinical article. J. Neurosurg. 115(2), 248-255 (2011). of antigen coupled to adjuvant to host APC. Although this immunotherapeutic strategy has met with only modest success in glioma patients to date, it is possible that ATC vaccines could be markedly more effective if their full power is unleashed by checkpoint blockade.

\section{Financial \& competing interests disclosure}

The authors have no relevant affiliations or financial involvement with any organization or entity with a financial interest in or financial conflict with the subject matter or materials discussed in the manuscript. This includes employment, consultancies, honoraria, stock ownership or options, expert testimony, grants or patents received or pending, or royalties.

No writing assistance was utilized in the production of this manuscript.

11 Okada H, Lieberman FS, Edington HD et al. Autologous glioma cell vaccine admixed with interleukin- 4 gene transfected fibroblasts in the treatment of recurrent glioblastoma: preliminary observations in a patient with a favorable response to therapy. J. Neurooncol. 64(1-2), 13-20 (2003).

12 Okada H, Lieberman FS, Walter KA et al. Autologous glioma cell vaccine admixed with interleukin-4 gene transfected fibroblasts in the treatment of patients with malignant gliomas. J. Transl. Med. 5, 67 (2007).

13 Parney IF, Chang LJ, Farr-Jones MA, Hao C, Smylie M, Petruk KC. Technical hurdles in a pilot clinical trial of combined B7-2 and GM-CSF immunogene therapy for glioblastomas and melanomas. J. Neurooncol. 78(1), 71-80 (2006).

14 Schneider T, Gerhards R, Kirches E, Firsching R. Preliminary results of active specific immunization with modified tumor cell vaccine in glioblastoma multiforme. $J$. Neurooncol. 53(1), 39-46 (2001).

15 Sobol RE, Fakhrai H, Shawler D et al. Interleukin-2 gene therapy in a patient with glioblastoma. Gene Ther. 2(2), 164-167 (1995).

16 Steiner HH, Bonsanto MM, Beckhove P et al. Antitumor vaccination of patients with glioblastoma multiforme: a pilot study to assess feasibility, safety, and clinical benefit. J. Clin. Oncol. 22(21), 4272-4281 (2004).

17 Vauleon E, Avril T, Collet B, Mosser J, Quillien V. Overview of cellular immunotherapy for patients with glioblastoma. Clin. Dev. Immunol. 2010, pii: 689171 (2010).

18 Bigner DD, Pitts OM, Wikstrand CJ. Induction of lethal experimental allergic encephalomyelitis in nonhuman primates and guinea pigs with human glioblastoma multiforme tissue. J. Neurosurg. 55(1), 32-42 (1981).

19 Volovitz I, Marmor Y, Azulay M et al. Split immunity: immune inhibition of rat gliomas by subcutaneous exposure to unmodified live tumor cells. J. Immunol. 187(10), 5452-5462 (2011).

20 Odoardi F, Sie C, Streyl K et al. T cells become licensed in the lung to enter the central nervous system. Nature 488(7413), 675-679 (2012). 